\section{$\sum_{\text {CHEST }}^{\text {CUNIC }}$} published online only. To view please visit the journal online (http://dx.doi.org/10.1136/ thoraxjnl-2014-205773).

${ }^{1}$ Department of Respiratory Medicine, Royal Brompton Hospital, London, UK. ${ }^{2}$ The University of Manchester \& University Hospital of South Manchester NHS Foundation Trust, Manchester

\section{Correspondence to}

Dr James H Hull, Department of Respiratory Medicine, Royal Brompton Hospital, Fulham Road, London SW3 6HP, UK; j.hull@rbht.nhs.uk

Received 20 May 2014 Revised 9 July 2014 Accepted 11 August 2014 Published Online First 26 September 2014

\title{
Lesson of the month: rowing-induced laryngeal obstruction: a novel cause of exertional dyspnoea: characterised by direct laryngoscopy
}

\author{
B Panchasara, ${ }^{1}$ C Nelson, ${ }^{1}$ R Niven, ${ }^{2}$ S Ward, ${ }^{1}$ J H Hull ${ }^{1}$
}

\begin{abstract}
Exercise-induced laryngeal obstruction (EILO) is a key differential diagnosis for unexplained dyspnoea in athletes. The gold standard means for diagnosis of EILO is direct laryngoscopy, performed continuously, while an athlete undertakes the specific sport that precipitates their symptoms. This report provides the first descriptions of rowing-associated EILO in two competitive rowers presenting with unexplained dyspnoea and cough. The report describes the methodology and safety of the use of continuous laryngoscopy in the context of maximal rowing ergometry and the use of this technique as a therapeutic tool to provide biofeedback.
\end{abstract}

\section{INTRODUCTION}

Unexplained breathlessness and cough are common symptoms reported by athletes. ${ }^{1}$ These symptoms are often attributed to the diagnosis exerciseinduced bronchoconstriction (EIB), and yet it is now recognised that exertional dyspnoea in athletes is frequently explained by the development of a transient obstruction, occurring at the level of the larynx. ${ }^{2}{ }^{3}$ This condition, termed exercise-induced laryngeal obstruction (EILO), typically occurs during intense, competitive bouts of exercise and can explain why 'asthma-type' symptoms appear refractory to inhaled therapy. ${ }^{2}$

Technological advances now permit accurate characterisation of EILO using the technique of direct and continuous laryngoscopy during exercise. ${ }^{3}$ More specifically, a flexible nasendoscope is placed to allow uninterrupted visualisation of laryngeal movement and permit detection of abnormal laryngeal closure during exercise. Additionally, it presents a potential means for biofeedback, offering the opportunity for an athlete to visualise their own laryngeal movement during exercise.

To date, the technique has been used in treadmill and cycle-ergometer settings, ${ }^{3}$ however, we now describe continuous laryngoscopy employed during rowing ergometer exercise. The test was used to facilitate diagnosis of unexplained dyspnoea in a sport-specific setting and specifically to identify and characterise rowing-associated EILO. We also describe the first use of laryngoscopy performed continuously during exercise as a means of providing biofeedback in this context.

\section{CLINICAL CASES}

\section{Case 1}

A 20-year-old atopic university-level rower was referred with a 4-year history of paroxysmal attacks of dyspnoea associated with dysphonia, wheeze and tightness in her upper chest and throat. Her exertional symptoms were precipitated almost entirely during rowing training or in competitive bouts. On the basis of exertional dyspnoea and cough she was diagnosed as asthmatic and treated with inhaled corticosteriod and short-acting $\beta-2$ agonist for 3 years prior to referral.

Investigations demonstrated preserved spirometric indices; $\mathrm{FEV}_{1}$ at $4.17 \mathrm{~L}(112 \%$ predicted) and FVC 5.25 (125\% predicted) and a normal chest radiograph.

She proceeded to undergo continuous laryngoscopy during exercise, but with apparatus adapted for use with a rowing ergometer (demonstrated by control subject in figure 1 and online supplementary video file E1). Specifically, the larynx was visualised by placing a flexible fibreoptic nasendoscope superior to the laryngeal aperture and fixed in specialist headgear (figure 1). A small quantity of topical lidocaine gel $(1 \mathrm{~mL}$ of $2 \%)$ was used to prepare the nares. Thereafter, images were continuously recorded on a digital recording system while the athlete performed a progressive increase in $500 \mathrm{~m}$ split times.

Resting laryngoscopy images revealed features of laryngopharyngeal reflux change (i.e. moderate diffuse laryngeal oedema). Rowing exercise precipitated significant abnormal inspiratory-phase medial and inward collapse of the supraglottic structures, commensurate with her typical 'asthma' symptoms of wheeze, dyspnoea and cough (figure 2 and online supplementary video file E2). There was no change in $\mathrm{FEV}_{1}$ following the exercise bout. She was referred for physiotherapy to optimise her breathing control and laryngeal relaxation.

\section{Case 2}

A 22-year-old, non-atopic internationally competitive, female rower was referred with an 8-year history of exertional dyspnoea, upper chest discomfort and cough. Her symptoms were confined to rowing exercise and most prominent during competitive races and high-intensity ergometer sessions. She denied the presence of a loud audible wheeze and denied significant nasal symptoms or gastrooesophageal reflux. Prior trials of inhaled bronchodilator and anti-inflammatory therapy failed to improve her symptoms.

Her spirometric indices were in the supranormal range; $\mathrm{FEV}_{1}$ 4.7 $\mathrm{L}(125 \%$ predicted) and $\mathrm{FVC}$ $5.80,(134 \%$ predicted). The amplitude of the flow- 
Figure 1 Continuous laryngoscopy during exercise performed on a rowing ergometer. (A) One of the authors

(CN) demonstrating equipment setup

(B) schematic of equipment.
A

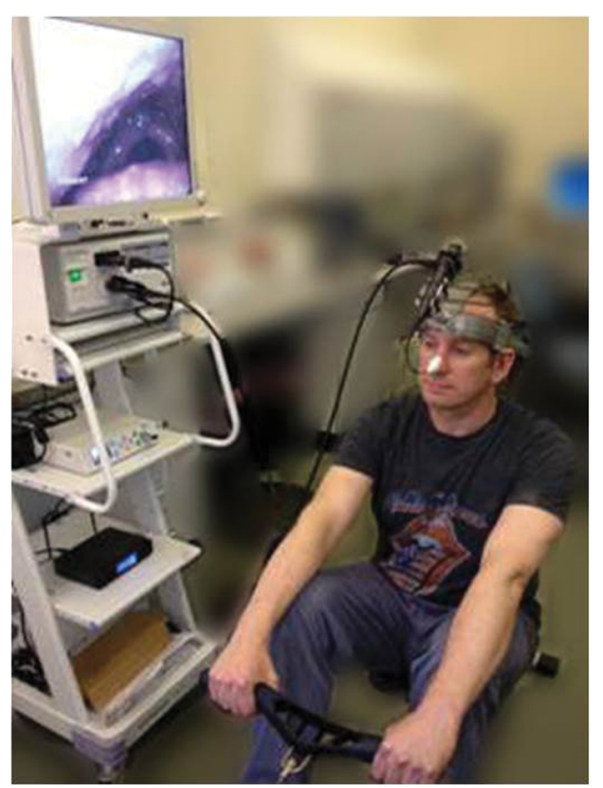

B

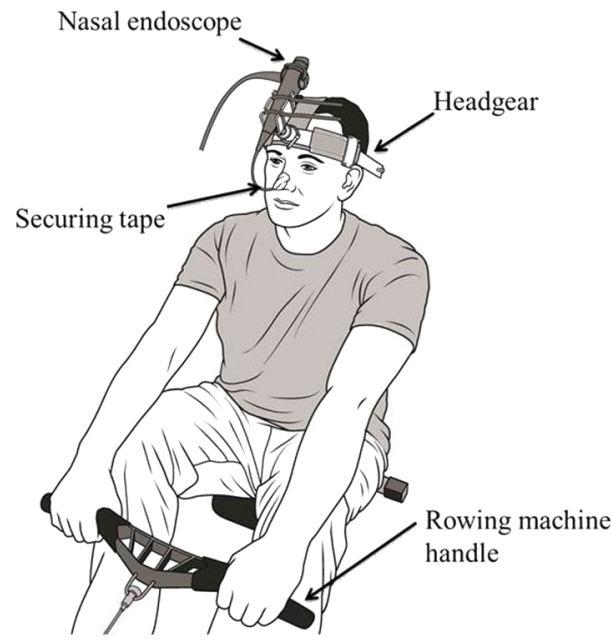

volume inspiratory flow limb was normal. A Mannitol bronchoprovocation test demonstrated no airways hyper-reactivity. Consequently, a diagnosis of vocal cord dysfunction was suspected and she was referred for further assessment.

She proceeded to undergoing rowing laryngoscopy, as described above. The test confirmed evidence of abnormal medial and inward rotation of the supraglottic structures in the inspiratory phase of breathing, although to a lesser extent than in the case above. This abnormal closure was associated with her 'typical' symptoms and a reduction in rowing pace; although she reported that the manifestations were only present at an intensity of approximately $50 \%$ that she typically develops when rowing on water.

Following the diagnostic session, time was spent, with the projection equipment visible to the athlete, in order for her to work on techniques to improve laryngeal control, i.e. biofeedback.

\section{DISCUSSION}

This report describes how it is possible to safely use exercise laryngoscopy in the context of intense rowing ergometry. This assessment permitted the acquisition of stable, high-quality images and successful characterisation of laryngeal movement during peak-intensity rowing exercise in order to diagnose rowing-associated EILO. In keeping with prior laryngoscopy testing reported in the literature, the rowing exercise-coupled laryngoscopy test was well tolerated and performed without complication or risk to the athlete. Moreover, it provides an opportunity for athletes to visualise their laryngeal closure in 'real-time', i.e. during rowing.
Figure 2 Image stills of laryngeal aperture taken during inspiration in the first patient; (A) rest, (B) moderate intensity exercise-slight internal rotation of arytaenoid structures, (C) peak exercise-partial occlusion of the larynx at the level of the supraglottis with abnormal adduction and medial rotation of supraglottic structures with tethering of aryepiglottic folds. This was commensurate with localising symptoms and reduction in rowing pace.

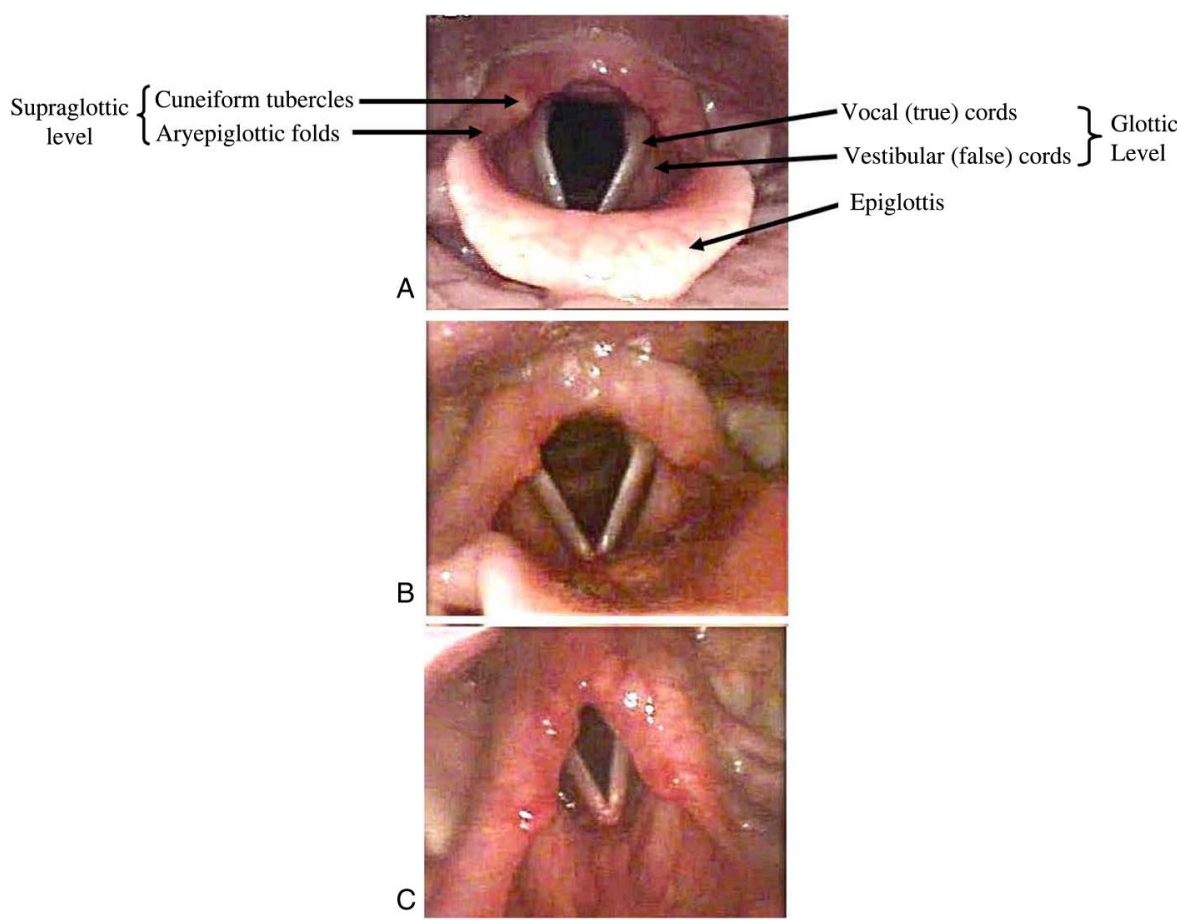


The larynx provides several vitally important functions and laryngeal closure permits an increase in intrathoracic pressure and, as such, is fundamental in several respiratory manoeuvres, including valsalva and cough. During an inspiratory manoeuvre, the neural discharge to the main laryngeal abductor muscle is closely aligned with contraction of the diaphragm. This close neurophysiological coupling is likely to be relevant in maintaining thoracic pressure during vigorous upper body exercise.

During exercise, there is evidence of a heightened activity of the laryngeal abductor muscles, acting to widen the laryngeal aperture, thereby minimising turbulence and airways resistance. Indeed, the normal response to exercise is for the laryngeal aperture to remain widely patent, to minimise resistance to flow.

In EILO, the laryngeal aperture narrows at peak exercise, typically secondary to an 'in-folding' of the supraglottic structures. ${ }^{2}{ }^{3}$ This may be followed by reduction in the diameter of the glottic aperture (i.e. vocal cord closure), however, the term, EILO, is favoured given that in the majority of cases closure occurs independent of vocal cord or fold adduction. It is postulated that this increases turbulence and creates the typical symptoms of wheeze, dyspnoea and cough. It is also likely to perpetuate a cycle of laryngeal irritability and, hence, may explain a propensity to cough.

To the best of our knowledge, laryngeal movement during intense competitive rowing exercise has never been studied, and thus, the 'normal' response is yet to be characterised. Rowing places specific demands on the ventilatory system, and control of intrathoracic pressure during certain sections of the rowing cycle is important. ${ }^{4}$ Further studies, with simultaneous measurement of laryngeal closure and respiratory flow and duty cycle impairment are required.

The reason why some athletes develop EILO is currently unclear. Several mechanisms have been postulated to explain development of EILO, and it is likely that laryngeal closure occurs as the result of a combination of factors. In this respect, the change in ventilation that occurs during rowing generates high airflow velocities over the laryngeal wall. Indeed as a group, elite rowers generate substantial increases seen in ventilation; up to $200 \mathrm{~L} / \mathrm{min}$ in men and $170 \mathrm{~L} / \mathrm{min}$ in women. This is likely to cause changes in pressure gradient across the larynx and potentiate the collapse of vulnerable laryngeal structures such as the aryepiglottic folds.

In women, differences in the structure of the larynx may predispose them to develop EILO. ${ }^{2}$ An aspect of psychological stress arising from intense competitive environments may also play a role in the development of laryngeal dysfunction. ${ }^{5}$

The optimum treatment for EILO has yet to be determined and prospective controlled studies are urgently needed to inform best management. The removal of laryngeal irritants (e.g. caffeine, untreated reflux) alongside breathing and laryngeal control techniques delivered by a physiotherapist or speech and language therapist form the cornerstone of therapy, however, surgical intervention has also been associated with good outcome. ${ }^{2}$ Inspiratory muscle training is an additional form of treatment which may provide benefit. ${ }^{4}$

During attendance, the second patient trialled a biofeedbackresponse style of therapy in which they viewed their larynx while rowing, in order to manipulate their breathing and maximise glottis opening. While previous studies ${ }^{5}$ have supported the use of biofeedback in gaining control over laryngeal dysfunction, this report is the first to report simultaneous endoscopic imaging of the larynx during rowing exercise. Prospective randomised studies are needed to assess the efficacy of biofeedback in this context.

In the cases reported, measurements were performed to rule out coexisting EIB, however, it is important to note that Nielsen and colleagues ${ }^{2}$ recently highlighted that EILO can coexist with EIB and thus, a diagnosis of EILO should not necessarily mandate cessation of inhaled therapy.

In conclusion, laryngoscopy performed during rowing exercise provides a safe and effective way of evaluating exercise-induced respiratory symptoms in rowers, to detect and characterise rowing-associated EILO. The latter should be considered in rowers presenting with unexplained dyspnoea and 'airway-centric' symptoms to avoid misdiagnosis of EIB and to facilitate a management strategy targeting improved laryngeal function.

Contributors JHH was responsible for the conception and design of the study; BP was responsible for drafting the manuscript. All remaining authors revised and approved the manuscript for intellectual content before submission.

Competing interests None.

Patient consent Obtained.

Provenance and peer review Not commissioned; externally peer reviewed.

\section{REFERENCES}

1 Hull J, Ansley L, Robson-Ansley P, et al. Managing respiratory problems in athletes. Clin Med 2012;12:351-6.

2 Nielsen EW, Hull JH, Backer V. High prevalence of exercise-induced laryngeal obstruction in athletes. Med Sci Sports Exerc 2013;45:2030-5.

3 Heimdal JH, Roksund OD, Halvorsen T, et al. Continuous laryngoscopy exercise test: a method for visualizing laryngeal dysfunction during exercise. Laryngoscope 2006;116:52-7.

4 Griffiths LA, McConnell AK. The influence of inspiratory and expiratory muscle training upon rowing performance. Eur J Appl Physiol 2007;99:457-66.

5 Earles J, Kerr B, Kellar M. Psychophysiologic treatment of vocal cord dysfunction. Ann Allergy Asthma Immunol 2003;90:669-71. 